\title{
Effect of a novel two-desk sit-to-stand workplace (ACTIVE OFFICE) on sitting time, performance and physiological parameters: protocol for a randomized control trial
}

\author{
Bernhard Schwartz ${ }^{1,2^{*}}$, Jay M. Kapellusch ${ }^{3}$, Andreas Schrempf ${ }^{1}$, Kathrin Probst ${ }^{4}$, Michael Haller ${ }^{4}$ and Arnold Baca ${ }^{2}$
}

\begin{abstract}
Background: Prolonged sitting is ubiquitous in modern society and linked to several diseases. Height-adjustable desks are being used to decrease worksite based sitting time (ST). Single-desk sit-to-stand workplaces exhibit small ST reduction potential and short-term loss in performance. The aim of this paper is to report the study design and methodology of an ACTIVE OFFICE trial.
\end{abstract}

Design: The study was a 1-year three-arm, randomized controlled trial in 18 healthy Austrian office workers. Allocation was done via a regional health insurance, with data collection during Jan 2014 - March 2015. Participants were allocated to either an intervention or control group. Intervention group subjects were provided with traditional or two-desk sit-to-stand workstations in either the first or the second half of the study, while control subjects did not experience any changes during the whole study duration.

Sitting time and physical activity (IPAQ-long), cognitive performance (text editing task, Stroop-test, d2R test of attention), workload perception (NASA-TLX) and physiological parameters (salivary cortisol, heartrate variability and body weight) were measured pre- and post-intervention (23 weeks after baseline) for intervention and control periods. Postural changes and sitting/standing time (software logger) were recorded th the workplace for the whole intervention period.

Discussion: This study evaluates the effects of a novel two-desk sit-to-stand workplace on sitting time, physical parameters and work performance of healthy office based workers. If the intervention proves effective, it has a great potential to be implemented in regular workplaces to reduce diseases related to prolonged sitting.

Trial registration: ClinicalTrials.gov Identifier: NCT02825303, July 2016 (retrospectively registered).

Keywords: Postural changes, Standing, Sitting, Cognitive performance, Reaction time, Concentration, Workload, Office, Stroop-test, d2R-test of attention

\section{Background}

Prolonged sitting is ubiquitous in modern society and the amount of physically inactive people is rising in many countries $[1,2]$. Ongoing computerization is a main cause for changes in physical activity and sitting time patterns $[3,4]$. Screen time, which is commonly

\footnotetext{
* Correspondence: bernhard.schwartz@univie.ac.at

1 Department of Medical Engineering, University of Applied Sciences Upper Austria, Garnisonstrasse 21, 4020 Linz, Austria

${ }^{2}$ Department of Sport Science, University of Vienna, Auf der Schmelz 6, 1150

Vienna, Austria

Full list of author information is available at the end of the article
}

associated with sitting, has been dramatically increased by a rising prevalence of computers in school and occupational environments [4]. Duration of sitting time has also been shown to increase with age [5].

In 2013, $11 \%$ of all European citizens (aged 14 years and older) spent more than $8.5 \mathrm{~h}$ per day in a sitting posture [6]. In the working age population, white-collar workers are most frequently affected by this amount of sitting time $(21 \%)$ and exhibit a more than four times higher risk of being exposed to prolonged sitting in comparison to manual occupations [6]. Especially office 
workers and call center employees are affected by prolonged sitting periods. The total amount of sitting in these occupations can exceed more than $80 \%$ of the working day $[7,8]$.

Prolonged sitting is a risk factor for cardiovascular and musculoskeletal diseases, diabetes, several types of cancer and all-cause mortality [9-14]. In combination with static and awkward postures, the prevalence of musculoskeletal diseases (e.g. back pain, chest pain) can increase further [14]. As additional physical activity cannot fully compensate the effects of prolonged sitting $[15,16]$, standing between prolonged sitting periods and reduction of sedentary pursuits should be a goal for adults, irrespective of their exercise habits [17].

Given that most of the world's population spend averagely one third of their adult life at work [18], it seems clear that worksite based interventions for reducing sitting time are key elements of daily sitting time reduction. Generally, worksite based recommendations in offices contain "Sit less", "Stand up", "Move more" and "Change postures regularly" [16, 19, 20]. In order to fulfill postural recommendations different types of worksite based interventions have been started. Besides numerous activity promotion programs, which typically replace sitting time with low-intensity physical activity [21], the implementation of sit-to-stand or active workstations is commonly used to diminish occupational sitting time [22].

Large differences in sitting time reduction have been found for different types of sit-to-stand workstations [22]. While non-significant changes in sitting time for sit-to-stand desk users in open plan offices occurred [23], meta-analysis showed an average reduction in sitting time of $77 \mathrm{~min}$ per 8-h workday for activitypermissive workstations [22]. Multi-component interventions (e.g. management consultation) can further enhance this effect [24].

Although the implementation of sit-to-stand or active workstations can help to reduce sitting time, improve physical activity at work and promote health benefits [25-27], it might also lead to changes in cognitive functions such as productivity [22]. Even though nonsignificant changes in attention have been found [28], fine motor functions (e.g. mouse moving) as well as mathematical problem solving can be negatively influenced by additional body movements [29]. As studies reporting deterioration of work-related outcomes were all of short duration, studies using long-term follow-up were recommended [22].

The occupational hazards associated with prolonged sitting are receiving renewed attention, and new technologies, devices, and workplace controls to help reduce sitting time are being developed and introduced regularly. It would benefit researchers, practitioners, and employers if these devices and controls were evaluated and studied using consistent and reproducible methods. Reliable and comparable information produced from similarly designed studies would help to separate fact from fiction in the efforts to reduce chronic sitting in the workplace.

\section{Objectives}

The primary objective of this paper is to describe and discuss the methods of a study designed to evaluate the long-term effect of a novel two desk sit-to-stand workplace on sitting time as well as physiological and cognitive parameters for healthy people of working age in comparison to their traditional workplace (control). A secondary objective is to propose methods for future studies of sit-to-stand equipment and intervention programs.

\section{Hypothesis}

The primary hypothesis of the described study is that the ACTIVE OFFICE two-desk sit-to-stand workplace are more effective in reducing occupational sitting time than conventional one desk solutions. Secondary hypotheses are that people using the ACTIVE OFFICE setup will experience positive long term effects on physiological and cognitive skills. The experimental groups received a two desk sit-to-stand workstation in their regular office environments.

\section{Methods/Design}

ACTIVE OFFICE is a three-arm randomized control trial with two intervention and one control group (Fig. 1). After the baseline assessment was completed, the participants were randomly allocated to either the intervention or the control arm in a 2:1 ratio. The experimental group subjects received a novel two desk sit-to-stand workstation in their regular office environments, while the control group subjects did not encounter any change in their regular office environments. A 6-week wash out phase was implemented to encourage similar starting conditions for each participant (i.e. using a traditional workplace prior to pre-intervention measurements).

\section{Participants}

A convenience sample of participants was recruited from companies in Linz (Austria) and the surrounding area. A general letter requesting collaboration and providing study descriptions was sent to employers. To reduce recruiting bias, partner-company allocation was randomly done via a regional health insurance provider between August and September 2013. Study details were provided to companies that accepted collaboration in the form of information seminars located at the respective company sites. Separate interviews with people 


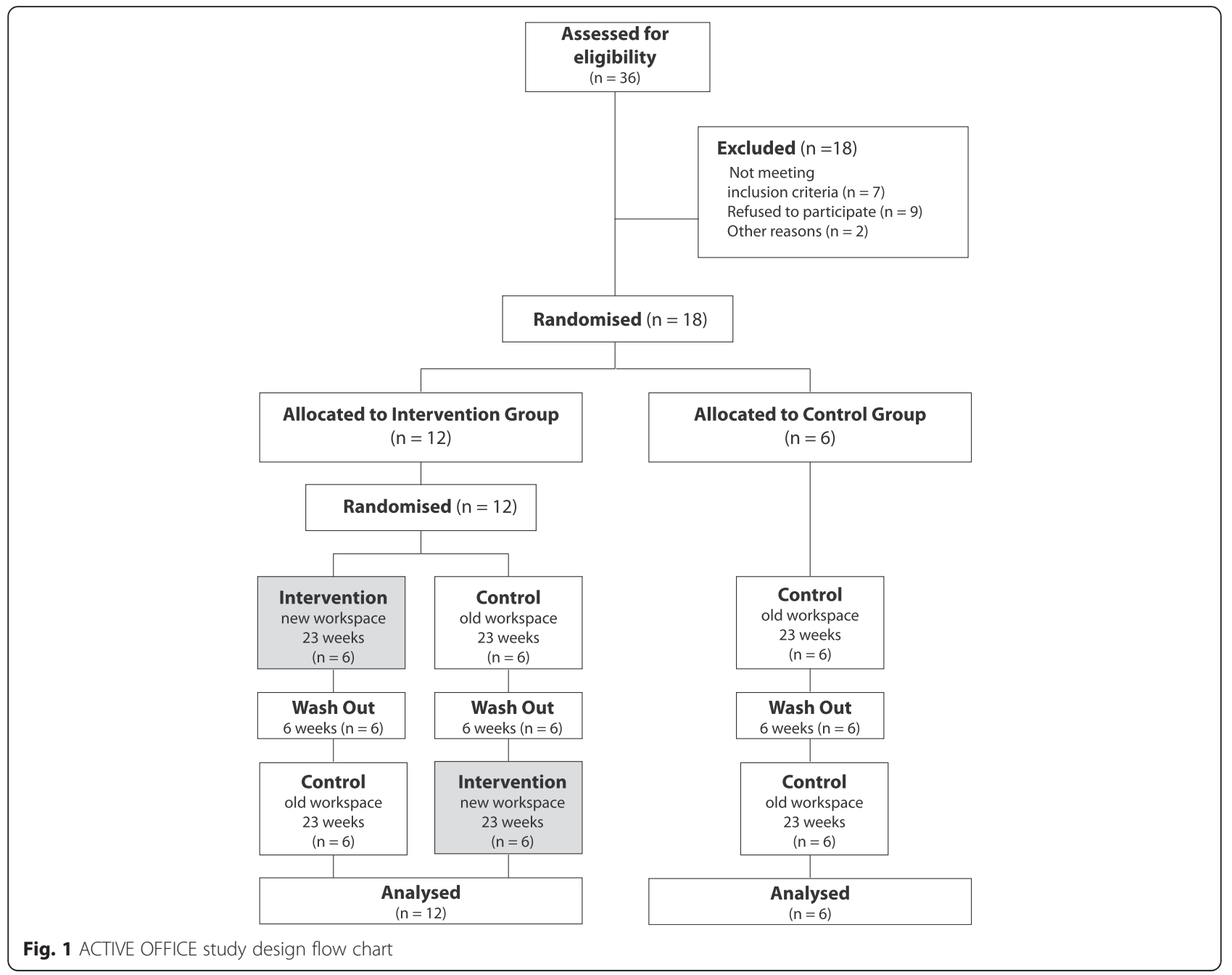

interested in participating in the study took place after the seminars to ascertain the potential subjects' suitability for study purposes. After exclusion criteria were applied, participants were allocated randomly either to intervention or control groups.

\section{Subject inclusion criteria}

Included subjects were: a) healthy caucasian (no acute or chronic diseases); b) normal weight or slightly overweight (BMI: $18.5-27.5 \mathrm{~kg} / \mathrm{m}^{2}$ ); c) aged: $18-60$ years; d)regularly working in sedentary office environments; e) regular computer users; f) fluent German speakers; and g) consented to participate.

\section{Subject exclusion criteria}

Excluded subjects had or were: a) heavily overweight \& Obesity (BMI $>27.5 \mathrm{~kg} / \mathrm{m}^{2}$ ); b) short office stay duration $(<8 \mathrm{~h} /$ day or $<20 \mathrm{~h} /$ week) c) experience in sit-tostand workstations; d) acute or chronic diseases; e) inability to stand; f) visual impairments that had not been corrected; g) color blindness h) women who are pregnant or plan to become pregnant within 12 months; i) people planning to change their physical activity level; j) regular smokers (> 1 cigarette /day); or k) not consented to participate.

\section{Randomization and blinding}

After the baseline assessment was completed, the participants were randomly allocated to either the intervention or the control (no intervention) arm in a 2:1 ratio (Fig. 1) by means of a covariate adaptive randomization [30]. Based on previous findings [31], 'company' has been determined as a stratum and thus participants were balanced across companies (i.e., 3 participants for each company). On a second level, intervention participants were assigned to either the first (intervention first) or the second (control first) intervention group. Due to the nature of the intervention, participants were not blind to their allocation. 


\section{Sample size}

A pilot study with 5 participants, performed in order to estimate the potential of the two-desk setup, found a 22 $\%$ reduction of sitting time [32]. Converted to a regular 8-h work day this results in 105 min of sitting time reduction. As this effect was noticeably higher than the effect shown by existing meta-analysis [22] we decided to detect a value between those limits. Therefore, 12 subjects would be needed to detect 90 min differences in sitting time, assuming an alpha risk of 0.05 and beta risk of 0.20 in a two-sided test, and with $20 \%$ loss to follow-up.

\section{Screening}

Study eligibility was determined in private interviews prior to the study. Age, body weight, stature, gender, physical and mental well-being, smoking habits, chronic and acute complaints, pregnancy, medical limitations, medication, working hours per day and week, main occupation and company affiliation were collected via a self-administered questionnaire.

\section{Intervention for experimental Group}

Figure 2 shows the ACTIVE OFFICE two desk sit-tostand intervention setup. It consists of two equal heightadjustable desks standing next to each other. Precise table arrangements (e.g. 90, 135 and $180^{\circ}$ ) were selfdetermined by the participants. To ensure equal conditions, every desk was furnished with the same amount and style of mice, keyboards and screens. Depending on their pre-intervention working conditions, the participants used either one or two screens per desk. The ACTIVE OFFICE was installed 1 day prior to the intervention period at the location of the old desk. Together with the study leader, desk heights were adjusted to the desired sitting and standing heights. Additional software tracking hardware inputs on the

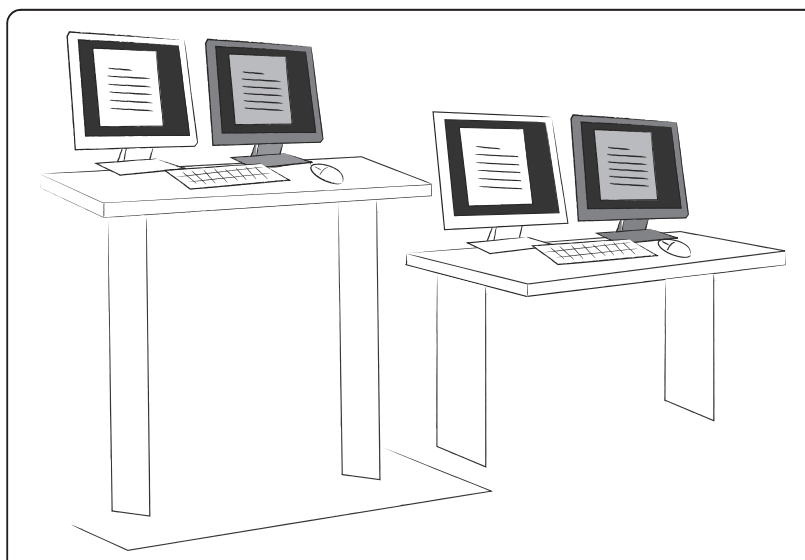

Fig. 2 ACTIVE OFFICE two desk sit-to-stand intervention setup standing or sitting desk were installed. The traditional desks were moved to storerooms at the local facility for the duration of the intervention period. During the control phase for the experimental group, both desks were fixed to the sitting height to simulate regular sitting environments. This strategy was used to reduce reconstruction work efforts when the experimental group switched from intervention to control, or vice versa. Reasonable care was been taken to ensure that both desks were equally furnished to avoid preferential effects during both the intervention and control phases (e.g. comparable construction and style of desk, identical equipment and furnishings).

\section{Control group}

Control group subjects did not encounter any changes in their regular office environments.

\section{Outcome measures}

Measurements were made both in the field and in a laboratory. Field measurements were made and processed continuously over the 23-week intervention period. Laboratory measurements were made on two different days, 1 day prior to intervention, and 1 day following intervention (due to cross-over design, each subject underwent 4 total days of laboratory measurements). Field measurements were collected automatically at the participants' workstation in their working office. Laboratory tests were conducted in a controlled, simulated work-space located at the University of Applied Sciences Campus Linz.

\section{Outcomes}

Primary outcomes were changes in sitting time after 23 weeks in the experimental group compared with its own control period and the control group.

Secondary outcomes within the experimental group were changes in: reaction time, working speed, level of attention, workload perception, physical activity and postural changing pattern for the intervention phase as compared to the control phase.

Tertiary outcomes were changes in salivary cortisol level and heartrate variability (HRV) within the experimental group.

\section{Experimental group: field measurements}

Logging-software was installed on each participant's computer. By recognizing hardware (mouse, keyboard) inputs on either the sitting or standing desk, the software could determine the proportion of time that a subject was standing versus sitting during the 23-week intervention period. 


\section{Experimental group: laboratory measurements}

All laboratory measurements were made in a controlled laboratory at the campus site Linz of the University of Applied Sciences Upper Austria. Temperature, air flow, humidity, lighting conditions (artificial light only) and noise level were controlled and set to be consistent with the subjects' typical working environment.

Participants were asked to refrain from exercise, caffeine and alcohol and undue stress for $24 \mathrm{~h}$ prior to laboratory testing. Food intake $90 \mathrm{~min}$ prior to the experiment was prohibited. Subjects were instructed to pursue their usual professional activity in the morning, followed by a laboratory visit in the early evening. To avoid daily fluctuations on performance all measurements started between 1:30 and 2:45 pm.

During the laboratory measurements, subjects either stood or sat upright in an ergonomic office chair, according to the study protocol. Subjects were encouraged to work as fast and as accurately as they could. To ensure identical testing conditions between subjects and to not unduly influence physiological parameters such as salivary cortisol level or heart rate variability, subjects were required to minimize excessive movement (e.g. standing up during the sitting periods). During regular breaks subjects were allowed to visit the toilet. Minor body movements, which typically occur under normal working conditions, were allowed.

\section{Reaction time, attention \& working speed}

Physical efforts when performing standardized tests (e.g. standing or walking) can negatively influence cognitive parameters $[28,29]$. As studies reporting deterioration of work-related outcomes were all of short duration [19] and there are indications that these are caused by non-familiar working conditions, long-term effects on cognitive performance remain to be identified. Within the study protocol, three different performancerelated tests were implemented:

A digital text editing task encouraging participants to fill in spaces in an ergonomic guideline text for $10 \mathrm{~min}$ was used. A Stroop-Color-Word-Conflict (Stroop) test, used to measure selective attention and processing speed [33-35] as well as a "d2R-test of attention" (d2R), commonly used in the European area to determine concentration performance [36-38], were implemented.

The simplicity of the text editing task (that did not require any disciplinary knowledge) enabled working speed measurements and simulated typical low effort office work. The implemented digital Stroop-test version contained 190 congruent, incongruent and neutral tasks and required approximately $10 \mathrm{~min}$ to simulate long-lasting monotonous office screen work. The d2R-test was executed as a pen and paper version. Therefore, it enabled screen breaks during the test protocol and simulated paper-related office work.

The Stroop-test and the d2R-test are both characterized by a high test-retest reliability $(r=0.77-0.95)$ and do not require any specific previous knowledge except of rudimentary language skills [39, 40]. Normative values for the $\mathrm{d} 2 \mathrm{R}$-test are available for different countries [39].

\section{Workload perception}

Sit-to-stand workstations can evoke positive as well as negative associations [22]. While additional physical efforts caused by standing can lead to higher discomfort especially in the lower extremities (e.g. leg swelling) [22], novel working environments can improve mental wellbeing [41]. A common method to rate workload perception is the NASA-TLX questionnaire [42]. For reasons of simplicity and unmodified sensitivity [42], the short version of this questionnaire (RTLX), consisting of six major items, was used. Influences on workload perception based on unweighted items in the RTLX were negated due to the cross-over design.

\section{Salivary cortisol level}

Although there are new findings related to the metabolic risks associated with postural changes (breaks in sedentary time) $[16,43,44]$, the effect on stress-related parameters is still unclear. Modified cortisol levels after implementing a novel workplace have been shown but the effect of postural changes on cortisol level is not yet known [41]. Therefore, salivary cortisol level was measured during the study protocol and on the following morning in order to detect the cortisol awakening response (CAR) [45].

\section{Heartrate variability}

Heartrate variability (HRV) can be used for predicting all-cause mortality and characterizing cardiovascular health [46, 47]. Improvements of HRV caused by additional physical effort have been shown mainly in physical training programs with medium or vigorous intensity [48-50]. Additional weekly metabolic efforts around 1000 METmin at low intensity level (walking) have also demonstrated positive changes in HRV [51]. Since additional standing (caused by occupational sitting time reduction) should lead to the same level of physical effort (assumption 20-30 \% standing), any effect on HRV would be detectable. The 30 min breaks within the study protocol as well as nocturnal periods were used to compare HRV under bias reduced conditions. According to the HRV guideline [46], $24 \mathrm{~h}$ Holter monitoring measurements have been implemented. HRV will be analyzed using the software Kubios [52]. 


\section{Controlling for outside of work physical activity and sedentary behavior}

Physical activity and sedentary behavior are related to physiological and cognitive changes [53, 54]. To avoid bias these parameters have to be determined. The International Physical Activity Questionnaire (IPAQ) has been shown to be reliable and valid for estimating physical activity and sitting time without any further measuring device [55-57]. The long version of this questionnaire (IPAQ-long) additionally enables it to distinguish between occupational and non-occupational activity. To adjust for outside of work sedentary behavior and/or physical activities, the IPAQ-long was interview administered at the beginning of each laboratory measurement day.

\section{Body movements}

Body movements can alter physiological parameters and cognitive performance $[28,58]$. Especially small movements during longer time intervals are very hard to classify my means of personal observations. Therefore, a three-dimensional accelerometer - placed on the sternum via a neoprene breast belt - was used to objectively measure body movements. Upper body placements of accelerometers have been shown to reliably detect body movements, and sit-to-stand as well as stand-to-sit transitions $[59,60]$. To reduce the total number of sensors, a HRV-recorder with integrated 3D-accelerometer was used (model: medilog AR12 plus, Schiller AG, Baar, Switzerland).

\section{Measurement protocols}

To test the study hypotheses, several parameters were defined and/or measured under standardized (laboratory measurements) and real life (field measurements) conditions (Table 1). Whereas body postures as well as postural changes were collected continuously during the 23-week intervention period, all further parameters were selectively measured before and after the intervention. To guarantee similar test sequences for each participant, a study protocol for laboratory measurements was developed (Fig. 3), consisting of three phases collecting physiological and cognitive parameters.

In the first (initial) phase, participants were familiarized with the study protocol. Sitting time and weekly physical activity were determined via the IPAQquestionnaire. Examples of each cognitive test implemented in the cognitive phase were executed according to their guidelines [39]. A $30 \mathrm{~min}$ break in a sitting posture was used to ascertain baseline heart-rate and cortisol level. Baseline heart-rate was calculated after a $20 \mathrm{~min}$ rest for a $5 \mathrm{~min}$ interval and saliva samples were collected at the end $(30 \mathrm{~min})$ of the break.

In the second (cognitive) phase subjects participated in a test battery containing five blocks. Each block consisted of a working speed test (text editing task), an attentional test (d2R-test of attention) and a reaction time test (Stroop-test). These tests lasted for $30 \mathrm{~min}$ to fulfill recommendations regarding postural changes [24]. To simulate "common" working conditions (computer based and non-computer based tasks), digital

Table 1 Parameters measured within the ACTIVE OFFICE study

\begin{tabular}{|c|c|c|c|c|c|c|}
\hline \multicolumn{3}{|l|}{ Measurement } & \multicolumn{2}{|l|}{ Location } & \multirow{2}{*}{$\begin{array}{l}\text { Data points } \\
d^{-1} \text { (overall) }\end{array}$} & \multirow{2}{*}{$\begin{array}{l}\text { Sampling rate } \\
\mathrm{s}^{-1}\end{array}$} \\
\hline Parameter & Method & Performed by & Laboratory & $\overline{\text { Office }}$ & & \\
\hline \multicolumn{7}{|l|}{ Physiological } \\
\hline Sitting time & IPAQ-long & questionnaire - interview & $x$ & & $1(4)$ & n.a. ${ }^{d}$ \\
\hline Physical activity & IPAQ-long & questionnaire - interview & $x$ & & $1(4)$ & n.a. ${ }^{d}$ \\
\hline Mental workload & NASA-TLX & questionnaire & $x$ & & $1(4)$ & n.a. ${ }^{d}$ \\
\hline Salivary cortisol & saliva collection & Salivette + cortisol ELISA & $x^{a}$ & & $8(32)$ & n.a. ${ }^{d}$ \\
\hline Heart-rate & ECG & ECG recorder & $x^{b}$ & & n.a. ${ }^{c}$ & 250 \\
\hline Body movements & acceleration & ECG recorder & $x^{b}$ & & n.a. ${ }^{c}$ & 250 \\
\hline \multicolumn{7}{|l|}{ Cognitive } \\
\hline Working speed & text editing task & computer software (matlab) & $x$ & & $5(20)$ & $>1000$ \\
\hline Reaction time & Stroop-test & computer software (matlab) & $x$ & & $5(20)$ & $>1000$ \\
\hline Attention & d2R-test & test sheet & $x$ & & $5(20)$ & n.a. ${ }^{d}$ \\
\hline \multicolumn{7}{|l|}{ Office based } \\
\hline Body postures & logging tool & computer software (C\#) & & $x$ & n.a. ${ }^{c}$ & $>1000$ \\
\hline Postural changes & logging tool & computer software (C\#) & & $x$ & п.а. ${ }^{c}$ & $>1000$ \\
\hline
\end{tabular}

${ }^{a}$ Seven measurements in the laboratory followed by one measurement at home on the following morning

${ }^{\mathrm{b}}$ Measurement starts in the laboratory and ends at home on the following morning

'Data points depending on duration of measurement

${ }^{\mathrm{d}}$ Non-digital measuring method (pen \& paper) 


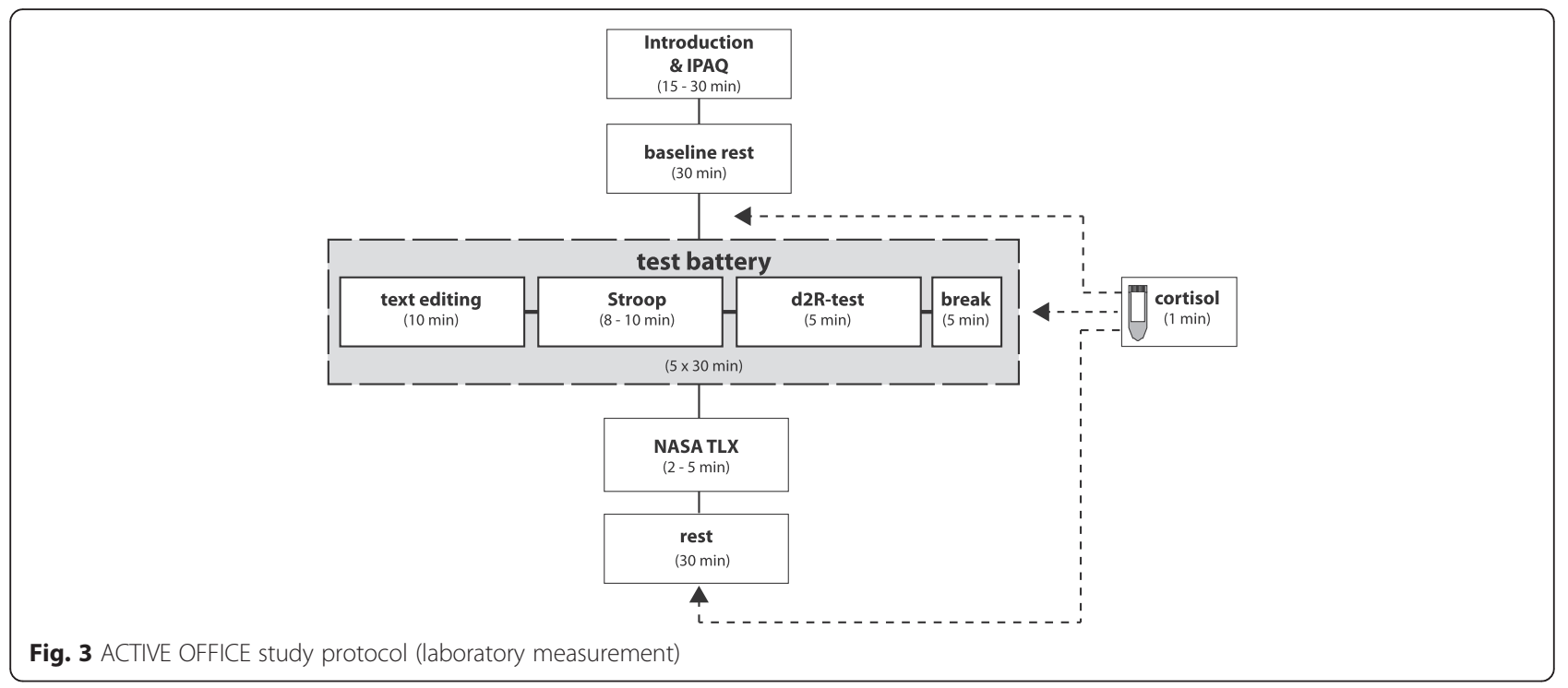

(text editing task, Stroop-test) as well as pen \& paper (d2R-test) versions of the implemented tests were used. All blocks were executed in alternating postures (sit - stand - sit - stand - sit) and at the end of each block - after a 5 min break - salivary samples were collected. The order of posture was not changed within groups or time.

In the third (final) phase participants were asked to estimate their workload by means of the NASA-TLX questionnaire followed by a $30 \mathrm{~min}$ resting phase in a sitting posture. During both $30 \mathrm{~min}$ resting phases (initial \& final) participants watched documentaries and were encouraged not to talk.

Salivary samples were collected after each break during the study protocol and on the following morning, 20 min after waking up, to ascertain cortisol awakening response (CAR). Salivary samples were centrifuged and stored at $-80{ }^{\circ} \mathrm{C}$ for subsequent testing using a chemiluminescent immunoassay.

Heart-rate was measured from the start of the study protocol until the CAR measurement.

\section{Statistical analysis}

Statistical analyses will be conducted using SPSS version 21 for windows (SPSS, Chicago, IL). Standard statistical methods will be used for calculations for means and standard deviations. Pre- and post- intervention differences will be calculated for sitting time and physical parameters. Paired t-tests will be used to show differences between pre- and post- conditions when the normality condition is satisfied. If not, Mann-Whitney-U tests will be used for pre-post comparison. For cognitive parameters, ANOVA with repeated measures will be used to test whether the different conditions have any effects on the outcome parameters assessed. To reduce learning effects the first block of the test-battery will be ruled out for analysis. When appropriate, post-hoc analyses will be conducted. The effects of time, group and interaction between both variables will be evaluated. To test for normality and homogeneity of variance, Shapiro-Wilk-test and Levene-test will be used, respectively. In general, two-sided tests with an alpha risk of 0.05 and a beta risk of 0.2 are to be accepted.

\section{Discussion}

The ACTIVE OFFICE study evaluates the effects of a novel two desk sit-to-stand workplace on occupational sitting time for healthy office workers. Secondary and tertiary outcomes will deliver insights in physiological and performance-related changes. To our knowledge, a workplace intervention consisting of two equally furnished height-adjustable desks has not been investigated to date.

This study design and approach has several strengths, including the randomized controlled trial design, statistical power analysis, strong inclusion criteria, identical environmental measurement conditions and objective assessment of the primary outcome based on a pilot study.

The study includes recruitment of several different companies to convey a greater pool of people with ergonomic ideas and provide insights into typical Austrian office workplaces. The resulting multisite bias has been reduced by a randomization stratum. The study's inclusion criteria support a homogeneous collective and will fortify findings. The robust nature of this study design is expected to provide insights into benefits of a two desk sit-to-stand setup. These methods could be employed to study other specific sit-stand interventions or strategies in a robust way. 
There are some noteworthy limitations of this study design. For example, as a result of the nature of the intervention it was not possible to blind subjects, although the researcher responsible for the statistical analyses will be blinded. Another limitation is the repeatability of the implemented cognitive tests. Although evaluations regarding short-term reliability have been executed [39, 61], the learning effect resulting from multiple repetition of the "d2R-test of attention" is unknown. Furthermore, as the implemented tests were evaluated in sitting postures only [39], the short-term effect of alternating postures on the performance (e.g. less performance caused by unfamiliar working posture) creates an additional bias. To reduce this bias, a shortterm study implementing the ACTIVE OFFICE study protocol has been performed, but data have not yet been analyzed.

There are some additional limitations that are specific to the ACTIVE OFFICE study but these could be easily overcome for future studies. First, due to limitations of hardware input detection, worker idle time (e.g. reading a document or leaving the workstation for a break) could not be directly measured. Sophisticated algorithms can be used to determine whether gaps between hardware inputs should be classified as sitting or standing, but these are imperfect (e.g. $1 \mathrm{~min}$ idle time between two sitting periods leads to the conclusion that the subject was sitting the whole time period). Hence, proximity sensors are proposed to be used in future studies to identify associated working postures and idle times more precisely.

Second, the sample size for the ACTIVE OFFICE study is small, and thus statistical power might be limited. Researchers will be able to use the forthcoming results of the ACTIVE OFFICE study to determine appropriate sample sizes for future studies.

This study design is intended to quantify the short to mid-term benefits of using a sit-stand intervention device of strategy. As specified, the design cannot draw conclusions about the long-term sustainability of any measured differences in behavior or performance, nor any long-term health outcomes associated with the changes. Multi-year, prospective studies are needed to test the efficacy of sit-stand technologies, devices, and administrative strategies. Nevertheless, the study design described here provides a repeatable, minimally biased approach to determine what devices and/or strategies have the potential to alter worker behavior and provide positive health benefits.

If the ACTIVE OFFICE setup proves to be successful intervention, it has potential to be implemented in common workplaces. This is crucial since alternating postures as well as reduction in prolonged sitting can promote health benefits and prevent several diseases
[16, 20, 43, 44]. Healthy individuals will likely also exhibit less absence time (increase in performance) which in turn leads to decreased health care system costs. If cognitive performance improvements can be shown, additional costs for a two desk setup will become more acceptable.

The methods used for the ACTIVE OFFICE study are generalizable and can serve as a common foundation upon which future studies can determine the potential efficacy of sit-stand devices and strategies. If future studies employ substantially similar methods, the results between studies would likely be directly comparable and this would help employers, practitioners, and future researchers to design appropriate sit-stand interventions.

\section{Trial status}

The recruitment for the "ACTIVE OFFICE" trials was initiated during August - September 2013. The baseline measurements and the post intervention measurements (23 weeks after baseline) were completed in September 2014 and April 2015, respectively. The study is currently at the stage of data analysis.

\section{Acknowledgements}

The authors thank all participants involved in this research as well as the Upper Austrian health insurance (Oberoesterreichische Gebietskrankenkasse) for allocating them. They further thank Joseph Gloeckl (Aeris Impulsmöbel $\mathrm{GmbH} \&$ Co. KG) and Christian Ecker (Schiller Medizintechnik GmbH) for donating all height-adjustable desks and ECG-recorders used in this study. These companies had no role in the design, analysis or writing of this article.

\section{Funding}

This study is partly supported by the Austrian Research Promotion Agency (FFG, BRIDGE program, 834185, "ACTIVE OFFICE"). The funders had no role in the design, analysis or writing of this article.

\section{Availability of data and materials}

Data resulting from this study will be analyzed and published in the PIs' PhD-thesis and journal articles.

\section{Authors' contributions}

Corresponding and first author BS led the development of this manuscript. He contributed to the research design and collected and analyzed data. AS and $\mathrm{MH}$ initiated the project "ACTIVE OFFICE". KP developed the loggingsoftware and was part of the ACTIVE OFFICE team. AB and AS contributed to the research design and played a major role in the study implementation. Authors $A B$ and JK participated in the manuscript writing. All authors read and approved the final manuscript.

\section{Authors' information}

BS is a research associate at the University of Applied Sciences Upper Austria and a PhD-student at the University of Vienna. The study described in this article is part of his PhD-thesis.

\section{Competing interests}

The authors declare that they have no competing interests.

Consent for publication

Not applicable.

Ethics approval and consent to participate

The study was conducted in accordance with the principles of the Helsinki Declaration and followed Austrian's best practice guidelines (Good Scientific 
Practice). The protocol and all pertinent documents have been evaluated and approved by the Ethics Committee of the University of Vienna (Reference number: 00052). Data confidentiality was guaranteed in accordance to the Austrian law governing the protection of personal data (Bundesgesetz über den Schutz personenbezogener Daten (Datenschutzgesetz 2000 - DSG 2000), (165/1999 17 August)). All study participants have given written consent to participate prior to involvement in the study.

\section{Author details}

'Department of Medical Engineering, University of Applied Sciences Upper Austria, Garnisonstrasse 21, 4020 Linz, Austria. ${ }^{2}$ Department of Sport Science, University of Vienna, Auf der Schmelz 6, 1150 Vienna, Austria. ${ }^{3}$ Department of Occupational Science \& Technology, University of Wisconsin - Milwaukee, P.O. Box 413, Milwaukee, WI 53201, USA. ${ }^{4}$ Media Interactive Lab, University of Applied Sciences Upper Austria, Softwarepark 11, 4232 Hagenberg, Austria.

\section{Received: 24 June 2016 Accepted: 7 July 2016}

\section{Published online: 15 July 2016}

\section{References}

1. Lynch BM. Sedentary behavior and cancer: a systematic review of the literature and proposed biological mechanisms. Cancer Epidemiol Biomarkers Prev. 2010;19:2691-709.

2. World Health Organization. Global recommendations on physical activity for health. Switzerland: WHO Press; 2010. p. 1-60. ISBN 978-92-4-159997-9. http://www.who.int/dietphysicalactivity/publications/9789241599979/en/. Accessed 23 Sept 2015.

3. Church TS, Thomas DM, Tudor-Locke C, Katzmarzyk PT, Earnest CP, Rodarte RQ, Martin CK, Blair SN, Bouchard C. Trends over 5 decades in U.S. occupation-related physical activity and their associations with obesity. PLoS One. 2011:6:1-7.

4. Owen N, Sparling PB, Healy GN, Dunstan DW, Matthews CE. Sedentary behavior: emerging evidence for a new health risk. Mayo Clin Proc. 2010;85: $1138-41$.

5. Matthews CE, Chen KY, Freedson PS, Buchowski MS, Beech BM, Pate RR, Troiano RP. Amount of time spent in sedentary behaviors in the United States, 2003-2004. Am J Epidemiol. 2008;167:875-81.

6. Eurobarometer \& European Commission. Special Eurobarometer 412 "Sport and Physical Activity". 2014

7. Clark B, Thorp A, Winkler E, Gardiner P, Healy G, Owen N, Dunstan D. Validity of self-report measures of workplace sitting time and breaks in sitting time. Med Sci Sport Exerc. 2011:43:1.

8. Straker L, Abbott RA, Heiden M, Mathiassen SE, Toomingas A. Sit-stand desks in call centres: Associations of use and ergonomics awareness with sedentary behavior. Appl Ergon. 2013;44:517-22.

9. Brown WJ, Miller YD, Miller R. Sitting time and work patterns as indicators of overweight and obesity in Australian adults. Int J Obes Relat Metab Disord. 2003;27:1340-6.

10. Van der Ploeg H, Chey T, Korda R, Banks E, Bauman A. Sitting time and all cause mortality risk in 222,497 Australian adults. J Sci Med. 2012:15:81-7.

11. Gierach GL, Chang S-C, Brinton L a, Lacey JV, Hollenbeck AR, Schatzkin A, Leitzmann MF. Physical activity, sedentary behavior, and endometrial cancer risk in the NIH-AARP Diet and Health Study. Int J Cancer. 2009;124:2139-47.

12. Patel AV, Rodriguez C, Pavluck AL, Thun MJ, Calle EE. Recreational physical activity and sedentary behavior in relation to ovarian cancer risk in a large cohort of US women. Am J Epidemiol. 2006;163:709-16.

13. Peeters G, Burton NW, Brown WJ. Associations between sitting time and a range of symptoms in mid-age women. Prev Med (Baltim). 2013;56:135-41.

14. Lis AM, Black KM, Korn H, Nordin M. Association between sitting and occupational LBP. Eur Spine J. 2007;16:283-98.

15. Healy GN, Dunstan DW, Salmon J, Shaw JE, Zimmet PZ, Owen N. Television time and continuous metabolic risk in physically active adults. Med Sc Sports Exerc. 2008;40:639-45.

16. Peddie MC, Bone JL, Rehrer NJ, Skeaff CM, Gray AR, Perry TL. Breaking prolonged sitting reduces postprandial glycemia in healthy, normal-weight adults: a randomized crossover trial. Am J Clin Nutr. 2013:98:358-66.

17. Garber CE, Blissmer B, Deschenes MR, Franklin B a, Lamonte MJ, Lee IM, Nieman DC, Swain DP. Quantity and quality of exercise for developing and maintaining cardiorespiratory, musculoskeletal, and neuromotor fitness in apparently healthy adults: guidance for prescribing exercise. Med Sci Sports Exerc. 2011;43:1334-59.
18. Alkhajah TA, Reeves MM, Eakin EG, Winkler EAH, Owen N, Healy GN. Sit-stand workstations. Am J Prev Med. 2012:43:298-303.

19. Healy GN, Eakin EG, LaMontagne AD, Owen N, Winkler EAH, Wiesner G, Gunning L, Neuhaus M, Lawler S, Fjeldsoe BS, Dunstan DW. Reducing sitting time in office workers: short-term efficacy of a multicomponent intervention. Prev Med (Baltim). 2013;57:43-8.

20. Dunstan DW, Howard B, Healy GN, Owen N. Too much sitting - a health hazard. Diabetes Res Clin Pract. 2012;97:368-76.

21. Conn VS, Hafdahl AR, Cooper PS, Brown LM, Lusk SL. Meta-analysis of workplace physical activity interventions. Am J Prev Med. 2009;37:330-9.

22. Neuhaus M, Eakin EG, Straker L, Owen N, Dunstan DW, Reid N, Healy GN. Reducing occupational sedentary time: a systematic review and meta-analysis of evidence on activity-permissive workstations. Obes Rev. 2014;15:822-38.

23. Gilson ND, Suppini A, Ryde GC, Brown HE, Brown WJ. Does the use of standing "hot" desks change sedentary work time in an open plan office? Prev Med (Baltim). 2012;54:65-7.

24. Neuhaus M, Healy GN, Dunstan DW, Owen N, Eakin EG. Workplace sitting and height-adjustable workstations: a randomized controlled trial. Am J Prev Med. 2014:46:30-40.

25. Koepp GA, Manohar CU, McCrady-Spitzer SK, Ben-Ner A, Hamann DJ, Runge CF, Levine JA. Treadmill desks: a 1-year prospective trial. Obesity. 2013;21:705-11.

26. Robertson MM, Ciriello VM, Garabet AM. Office ergonomics training and a sit-stand workstation: effects on musculoskeletal and visual symptoms and performance of office workers. Appl Ergon. 2013:44:73-85.

27. Elmer SJ, Martin JC. A cycling workstation to facilitate physical activity in office settings. Appl Ergon. 2014;45:1240-6.

28. Commissaris DACM, Könemann R, Hiemstra-van Mastrigt S, Burford E-M, Botter J, Douwes M, Ellegast RP. Effects of a standing and three dynamic workstations on computer task performance and cognitive function tests. Appl Ergon. 2014;45:1570-8.

29. John D, Bassett D, Thompson D, Fairbrother J, Baldwin D. Effect of using a treadmill workstation on performance of simulated office work tasks. J Phys Act Heal. 2009:6:617-24.

30. Kang M, Ragan BG, Park J-H. Issues in outcomes research: an overview of randomization techniques for clinical trials. J Athl Train. 2008:43:215-21.

31. Duncan MJ, Short C, Rashid M, Cutumisu N, Vandelanotte C, Plotnikoff RC. Identifying correlates of breaks in occupational sitting: a cross-sectional study. Build Res Inf. 2015;43:646-58.

32. Probst $K$, Lindlbauer D, Perteneder F, Schwartz B, Schrempf A. Exploring the use of distributed multiple monitors within an activity-promoting sit-andstand office workspace. In: Proceedings of the 14th IFIP TC13 Conference on Human-Computer Interaction. Volume 8119. Capetown; 2013:476-493.

33. MacLeod CM. The stroop task in cognitive research. In: Cognitive methods and their application to clinical research. Washington: American Psychological Association; 2005. p. 17-40.

34. Van der Elst W, Van Boxtel MPJ, Van Breukelen GJP, Jolles J. The Stroop color-word test: influence of age, sex, and education; and normative data for a large sample across the adult age range. Assessment. 2006:13:62-79.

35. Mead LA, Mayer AR, Bobholz J a, Woodley SJ, Cunningham JM, Hammeke TA, Rao SM. Neural basis of the Stroop interference task: response competition or selective attention? J Int Neuropsychol Soc. 2002;8:735-42.

36. Bates ME, Lemay EP. The $\mathrm{d} 2$ Test of attention: construct validity and extensions in scoring techniques. J Int Neuropsychol Soc. 2004;10:392-400.

37. Wassenberg R, Hendriksen JGM, Hurks PPM, Feron FJM, Keulers EHH, Vles JSH, Jolles J. Development of inattention, impulsivity, and processing speed as measured by the $\mathrm{d} 2$ Test: results of a large cross-sectional study in children aged 7-13. Child Neuropsychol. 2008;14:195-210.

38. Duschek S, Muckenthaler M, Werner N, Reyes del Paso GA. Relationships between features of autonomic cardiovascular control and cognitive performance. Biol Psychol. 2009;81:110-7.

39. Brickenkamp R, Schmidt-Atzert L, Liepmann D. Test d2-Revision Aufmerksamkeits- Und Konzentrationstest. Goettingen: HOGREFE Verlag für Psychologie; 2010.

40. Franzen MD, Tishelman AC, Sharp BH, Friedman AG. An investigation of the test-retest reliability of the stroop colorword test across two intervals. Arch Clin Neuropsychol. 1987;2:265-72.

41. Thayer JF, Verkuil B, Brosschot JF, Kampschroer K, West A, Sterling C, Christie IC, Abernethy DR, Sollers JJ, Cizza G, Marques AH, Sternberg EM. Effects of the physical work environment on physiological measures of stress. Eur J Cardiovasc Prev Rehabil. 2010;17:431-9. 
42. Hart SG. Nasa-task load index (NASA-TLX); 20 years later. Proc Hum Factors Ergon Soc 50th Annu Meet. 2006;50(9):904-908.http://humansystems.arc. nasa.gov/groups/tlx/tlxpublications.html.

43. Healy GN, Dunstan DW, Salmon J, Cerin E, Shaw JE, Zimmet PZ, Owen N. Breaks in sedentary time: beneficial associations with metabolic risk. Diabetes Care. 2008:31:661-6.

44. Larsen RN, Shaw J, Healy GN, Dunstan DW. Breaking up of prolonged sitting over three days sustains, but does not enhance, lowering of postprandial plasma glucose and insulin in overweight and obese adults. Clin Sci. 2015; 129:117-27.

45. Chida Y, Steptoe A. Cortisol awakening response and psychosocial factors: a systematic review and meta-analysis. Biol Psychol. 2009;80:265-78.

46. Guidelines. Guidelines Heart rate variability. Eur Heart J. 1996;17:354-81.

47. Thayer JF, Yamamoto SS, Brosschot JF. The relationship of autonomic imbalance, heart rate variability and cardiovascular disease risk factors. Int J Cardiol. 2010;141:122-31.

48. Routledge FS, Campbell TS, McFetridge-Durdle JA, Bacon SL. Improvements in heart rate variability with exercise therapy. Can J Cardiol. 2010;26:303-12.

49. Myllymäki T, Rusko H, Syväoja H, Juuti T, Kinnunen M-L, Kyröläinen H. Effects of exercise intensity and duration on nocturnal heart rate variability and sleep quality. Eur J Appl Physiol. 2012;112:801-9.

50. Buchheit M, Simon C, Piquard F, Ehrhart J, Brandenberger G. Effects of increased training load on vagal-related indexes of heart rate variability: a novel sleep approach. Am J Physiol Heart Circ Physiol. 2004;287:H2813-8.

51. Paschoal MA, Polessi EA, Simioni FC. Evaluation of heart rate variability in trained and sedentary climacteric women. Arq Bras Cardiol. 2008:90:74-9.

52. Tarvainen MP, Niskanen J-P, Lipponen JA, Ranta-Aho PO, Karjalainen PA. Kubios HRV-heart rate variability analysis software. Comput Methods Programs Biomed. 2014;113:210-20.

53. Rasberry CN, Lee SM, Robin L, Laris B, Russell L, Coyle KK, Nihiser AJ. The association between school-based physical activity, including physical education, and academic performance: a systematic review of the literature. Prev Med (Baltim). 2011;52 Suppl 1(April 2010):S10-20.

54. Best JR, Nagamatsu LS, Liu-Ambrose T. Improvements to executive function during exercise training predict maintenance of physical activity over the following year. Front Hum Neurosci. 2014;8(May):353.

55. Hagströmer M, Bergman P, De Bourdeaudhuij I, Ortega FB, Ruiz JR, Manios Y, Rey-López JP, Phillipp K, Von Berlepsch J, Sjöström M. Concurrent validity of a modified version of the International Physical Activity Questionnaire (IPAQ-A) in European adolescents: the HELENA Study. Int J Obes. 2008;32:S42-8.

56. Hagströmer M, Oja P, Sjöström M. The International Physical Activity Questionnaire (IPAQ): a study of concurrent and construct validity. Public Health Nutr. 2007;9:755-62.

57. Craig CL, Marshall AL, Sjöström M, Bauman AE, Booth ML, Ainsworth BE, Pratt M, Ekelund U, Yngve A, Sallis JF, Oja P. International physical activity questionnaire: 12-country reliability and validity. Med Sci Sports Exerc. 2003; 35:1381-95.

58. Hennig J, Friebe J, Ryl I, Krämer B, Böttcher J, Netter P. Upright posture influences salivary cortisol. Psychoneuroendocrinology. 2000;25(1):69-83.

59. Van Lummel RC, Ainsworth E, Lindemann U, Zijlstra W, Chiari L, Van Campen P, Hausdorff JM. Automated approach for quantifying the repeated sit-to-stand using one body fixed sensor in young and older adults. Gait Posture. 2013;38:153-6.

60. Godfrey A, Bourke AK, Olaighin GM, van de Ven P, Nelson J. Activity classification using a single chest mounted tri-axial accelerometer. Med Eng Phys. 2011;33:1127-35.

61. Gualtieri CT, Johnson LG. Reliability and validity of a computerized neurocognitive test battery, CNS Vital Signs. Arch Clin Neuropsychol. 2006;21:623-43.

\section{Submit your next manuscript to BioMed Central and we will help you at every step:}

- We accept pre-submission inquiries

- Our selector tool helps you to find the most relevant journal

- We provide round the clock customer support

- Convenient online submission

- Thorough peer review

- Inclusion in PubMed and all major indexing services

- Maximum visibility for your research

Submit your manuscript at www.biomedcentral.com/submit 